\title{
An Efficient Finite Element Method for Numerical Modeling of Shallow Water Wave Propagation
}

\author{
Farzan Rashidi ${ }^{1}$ \\ ${ }^{1}$ ECE Department, University of Hormozgan, Bandar-Abbas, Iran \\ ${ }^{1}$ Corresponding author's Email: f.rashidi@ece.ut.ac.ir
}

\begin{abstract}
To improve wide angle, bottom-interacting situation with complex boundaries in ocean acoustic wave propagation, in this paper the finite element method is used to discretize the continuous depth system of equations. Coupled linear Galerkin finite element method in association with parabolic equation model is applied for depth discretization of underwater wave equation. This discretization leads to equivalent system of ordinary differential equations (ODEs) that are applied over complex shapes covered by variable nature of surface and bottom boundaries. These ODEs are estimated by Crank-Nicolson approach. Simulation results show the capability of the proposed method in comparison with the physically behavior of underwater sound propagation and some numerical results of standard software. The obtained results demonstrate good agreement and efficiency of the proposed method.
\end{abstract}

Keywords: Finite element method, parabolic equation, underwater wave equation, shallow water, ordinary differential equations.

\section{Introduction}

Parabolic equation model as a powerful approach in engineering problems is used widely for the study of sound wave propagation in ocean acoustics [1]. By assuming that energy propagates nearly horizontally at speed close to shear or compressional speeds, a parabolic equation (PE) model is derived by factoring the hyperbolic underwater wave operator into product of incoming and outgoing operators and assuming that the outgoing component of pressure field dominates the incoming component [2]. The main reason of popularity of PE based methods is due to marching nature of parabolic equation numerical algorithms and their considerably improvement in computational cost and time [3].

By spreading modern acoustics projects, scientists in mathematics and engineering have been forced to use advanced approaches for simulating complex nature of physically environments. These attempts have been led to several numerical techniques in computational underwater acoustics
[4]. Among different numerical techniques only split step Fourier algorithm, implicit finite difference and finite element techniques have gained widespread use. Methods based on finite difference and finite element approaches are used for wide angle, bottominteracting situation environments. While, for long range, narrow angle propagation with negligible bottom interaction, split step based techniques efficiently are used [5]. The strong speed and density contrasts encountered at the water-bottom interface adversely affect the efficiency of the above mentioned computational techniques that for maintaining the efficiency of these algorithms, it requires to use excessively fine grid for range and depth segments [6]. Today, finite element method (FEM) has become the most popular and reliable method for solving complex physical environments. It can easily handle discontinuity of geometrical shapes as well as material discontinuity [7]. In this paper, we use standard PE model as representing sound wave equation. Then, by using capability of Galerkin finite element method based on linear shape function, we discretize the continues governing equation on each element. By using some 
linearization the boundary conditions are imposed to element matrices by variability of density and refraction coefficient on each element. After assembling these element matrices, final governing ODE of equations is solved by Crank-Nicolson approach. Furthermore, this coupling algorithm, finite element parabolic equation (FEPE) method can be applied at very wide angle propagation and very hard bottom interaction that our considered test problem consist severe contrast in bottom layer.

This paper is organized as follows: In first section, we give brief overview about PE algorithm. Section 2, derives the FEPE method and finally in section 4, numerical implementation of proposed method for some standard test problems corresponding shallow and water, are presented.

\section{Parabolic Approximation}

In this section, we derive the parabolic equation model for range independent medium bounded above by a free surface at $z=0$ with a sound profile that supports large range propagation (for $r \rightarrow \infty$ ). We consider two dimension pressure field $p(z, r)$ where, $\mathrm{z}$ is depth and the range $\mathrm{r}$ is the horizontal distance from a source that located at $\left(z_{S}, 0\right)$. By working in frequency domain, the factor $e^{-i \omega t}$ is removed, where, $\omega$ is the circular frequency and $t$ is time. For harmonic source in $r>0$, the pressure field satisfies at the following far-field equation,

$\frac{1}{r} \frac{\partial}{\partial r}\left(r \frac{\partial p}{\partial r}\right)+\rho \frac{\partial}{\partial z}\left(\rho^{-1} \frac{\partial p}{\partial z}\right)+k_{0}^{2} n^{2} p=0$

Where, $k_{0}=2 \pi f / c_{0}$ is the reference wave number, the notations $c(z), \rho(z)$ denote local sound speed, density [8]. By assuming the acoustic sound wave propagates along principle direction, the sound field can be separated as a slowly varying envelope and a fast oscillating phase term

$\psi(z, r)=\sqrt{k_{0} r} p(z, r) e^{-i k_{0} r}$

where, this envelope function, $\psi$, varies slowly with r. Substituting the above form into the equation (1) the wave equation for $k_{0} r \gg 1$, is split into two terms that governs the evolution of the forward and the backward sound wave of $\Psi$. By neglecting backward sound waves, the one-way equation can be obtained as follows,

$\frac{\partial \psi}{\partial r}=i k_{0}(Q-1) \psi$
Where,

$Q=\sqrt{1+X}$

and $X=k_{0}^{-2}\left(\rho \partial_{z}\left(p^{-1} \partial_{z}\right)\right)+\left(n^{2}(z)-1\right)$.

At the discontinuity interface, $\Gamma_{d}$, between two media having different density, the $\psi$ satisfies the following continuity condition,

$\frac{1}{\rho^{+}} \frac{\partial \psi^{+}}{\partial n}=\frac{1}{\rho^{-}} \frac{\partial \psi^{-}}{\partial n}, \psi_{z}^{+}=\psi_{z}^{+}$

Where, the superscript "+" (or "-") indicates that its associated quantities are on the "+" (or "-") side of $\Gamma_{d}$. We will not describe here the more details about underwater acoustic wave propagation models and refer readers to [9].

To solve equation (3), the square root operator $Q$ needs to be approximated. The standard approximation based on Taylor expansion is as:

$\sqrt{1+X} \cong 1+\frac{1}{2} X$

This yields the standard parabolic equation (SPE) which has been shown to be valid only for propagation angle $15-25^{\circ}$ of horizontal.

For one term only from the Pade series, the square root operator can be written as wide angle parabolic equation (WAPE) of Claerbout:

$\sqrt{1+X} \cong \frac{a_{0}+a_{1} X}{b_{0}+b_{1} X}$

Where, $a_{0}=1, a_{1}=\frac{3}{4}, b_{0}=0$ and $b_{1}=\frac{1}{4}$.

Any numerical method based on PE requires the acoustic field over depth at the initial depth $r_{0}$ that we use Gaussian starter as the following source function [10],

$$
\psi(0, r)=\sqrt{k_{0}} \exp \left(-\frac{k_{0}^{2}}{2}\left(z-z_{S}\right)^{2}\right.
$$

\section{Galerkin Finite Element Method}

The FEM is one of more powerful methods that have been gain widespread use in many branches of engineering problems. It is based on subdividing a complex shape into simple shapes which are much more mathematically manageable [11]. In this paper, 
this subdividing is done over depth interval $\left[0, z_{\max }\right]$ with $N_{z}$ linear elements $0=z_{0}<\cdots<$ $z_{N_{z}}=z_{\max }$. The nodes are denoted by $z_{i}, i=$ $0, \ldots, N_{z}$. Each linear element $i$ consists the domain $\left[z_{i-1}, z_{i}\right]$ that is denoted by $\Omega^{i}, i=1, \ldots, N_{z}$. The discrete equation for FEM is obtained from interplant functions to $\psi$ on each element $\Omega^{\mathrm{e}}$ for governing equation (3) in terms of shape functions that is restriction of basis functions to each element as:

$\psi^{e}(z, r)=\sum_{j=1}^{2} u_{j}^{e}(r) \phi_{j}(z)$

Where for an arbitrary range $r$, the function values $u^{e}$ are unknown parameters. The functions $\phi_{j}$ are defined as:

$\phi_{j}(z)=\left\{\begin{array}{cc}\frac{z-z_{j-1}}{h_{j}}, & z \in \Omega^{j-1} \\ \frac{z_{j}-z}{h_{j+1}}, & z \in \Omega^{j} \\ 0 & \text { else }\end{array}\right.$

Where, $h_{j}=z_{j}-z_{j-1}$ is the length of linear element $\Omega^{\mathrm{j}}$. These test functions satisfy the following properties.

1. Each test functions $\phi_{j}(z) \in H_{0}^{1}\left(\Omega^{\mathrm{j}}\right)$.

2. Each test functions $\phi_{j}(z)$ is piecewise over each element.

3. The $\phi_{j}\left(z_{i}\right)=\delta_{i j}$, where, $\delta_{i j}$ is Kronecker delta.

4. If $|i-j| \geq 2$ then $\phi_{i}\left(z_{j}\right)=0$,

Where, the $H^{1}\left(\Omega^{j}\right)$ is the Sobolev space that is defined as,

$$
\begin{gathered}
H^{\alpha}\left(\Omega^{\mathrm{j}}\right)=\left\{v \in L^{2}\left(\Omega^{j}\right): \frac{\partial^{i} v}{\partial z} \in L^{2}\left(\Omega^{j}\right),|i|\right. \\
\leq \alpha\}
\end{gathered}
$$

Where for $H^{1}\left(\Omega^{j}\right)$, we have the conditions, $v\left(z_{j-1}\right)=0$ and $v\left(z_{j}\right)=0$,

With the following inner product,

$$
(u, v)=\int_{\Omega^{j}} u v d \Omega^{j}, \quad u, v \in L^{2}\left(\Omega^{j}\right)
$$

In terms of local coordinate transformation the element $\Omega^{j}=\left[z_{j-1}, z_{j}\right]$ is mapped on $[0,1]$ by transformation $z=z_{j-1}+\xi h$ where, $0 \leq \xi \leq 1$. Thus the shape functions (11) are expressed as:

$\phi_{1}(\xi)=1-\xi$ and $\phi_{2}(\xi)=\xi$
After these preliminaries, we want to do the following steps:

1. Formulation of the governing equation (3) by variational technique,

2. Discretization of the continuous formulation,

3. Solving discretized system of equations.

For formulation step, we apply weak form of equation (3) with weight functions $W$ as follows,

$\int_{z_{j-1}}^{z_{j}}\left(\frac{\partial \psi}{\partial r}-i k_{0}(Q-1) \psi\right) W d z$

According to Galerkin method, both the weight function and approximate functions are chosen linear shape functions (9). Substituting $\mathrm{W}$ by $\phi_{k}(z)$ and $\psi$ by interpolate function $\psi^{e}$ leads to,

$$
\int_{z_{j-1}}^{z_{j}}\left(\frac{\partial \psi^{e}}{\partial r}-i k_{0}(Q-1) \psi^{e}\right) \phi_{k}(z) d z=0
$$

By employing interpolation equation (8), it obtains,

$$
\begin{aligned}
& \sum_{j=1}^{2} \frac{\partial u_{j}^{e}}{\partial r} \int_{z_{j-1}}^{z_{j}} \phi_{j}(z) \phi_{k}(z) d z \\
&-\frac{i k_{0}}{2} \sum_{j=1}^{2} u_{j}^{e} \int_{z_{j-1}}^{z_{j}}(Q \\
&-1) \phi_{j}(z) \phi_{k}(z) d z=0
\end{aligned}
$$

where, $k=1$,2. Substituting SPE (5) for environment depth operator $\mathrm{Q}$, second integral in above equation broken to following integrals on each element,

$\frac{1}{2 k_{0}^{2}} \int_{z_{j-1}}^{z_{j}} \rho \frac{\partial}{\partial z}\left(\frac{1}{\rho} \frac{\partial}{\partial z}\right) \phi_{j}(z) \phi_{k}(z) d z$,

And

$\int_{z_{j-1}}^{z_{j}}\left(n^{2}(z)-1\right) \phi_{j}(z) \phi_{k}(z) d z$

By using equations (18) and (19) and transforming all integrals in local coordinates with linearization in density and refraction coefficient, we obtain, 


$$
\begin{aligned}
& \sum_{j=1}^{2} \frac{\partial u_{j}^{e}}{\partial r} \int_{0}^{1} \phi_{j}(\xi) \phi_{k}(\xi) d \xi \\
& -\frac{i}{2 k_{0} h^{2}} \sum_{j=1}^{2} \tilde{\rho}^{e} u_{j}^{e} \int_{0}^{1} \frac{\partial \phi_{j}(\xi)}{\partial \xi} \frac{\partial \phi_{k}(\xi)}{\partial \xi} d \xi \\
& +i k_{0} \sum_{j=1}^{2} \tilde{n}^{e} u_{j}^{e} \int_{0}^{1} \phi_{j}(\xi) \phi_{k}(\xi) d \xi=0
\end{aligned}
$$

Where, for the values of density $\rho_{1}, \rho_{2}$ and refraction coefficients $n_{1}, n_{2}$ in the end points of each element, we have,

$\tilde{\rho}^{e}=\frac{2 \rho_{1}}{\rho_{1}+\rho_{2}} . \quad$ and $\tilde{n}^{e}=\frac{\left(\tilde{n}_{1}^{2}-1\right)+\left(\tilde{n}_{2}^{2}-1\right)}{4}$.

In matrix representation,

$A^{e} \frac{\partial u^{e}}{\partial r}+\left[\mu B^{e}+v C^{e}\right] u^{e}=0$

where, $\mu=\frac{i}{2 k_{0} h^{2}}, v=\frac{-i k_{0}}{h}$ and

$A_{j k}^{e}=\int_{0}^{1} \phi_{j}(\xi) \phi_{k}(\xi) d \xi=\frac{1}{6 h}\left(\begin{array}{ll}2 & 1 \\ 1 & 2\end{array}\right)$

$B_{j k}^{e}=\tilde{\rho} \int_{0}^{1} \frac{\partial \phi_{j}(\xi)}{\partial \xi} \frac{\partial \phi_{k}(\xi)}{\partial \xi} d \xi=\tilde{\rho}\left(\begin{array}{cc}1 & -1 \\ -1 & 1\end{array}\right)$

$C_{j k}^{e}=\tilde{n} \int_{0}^{1} \phi_{j}(\xi) \phi_{k}(\xi) d \xi=\frac{\tilde{n}}{6 h}\left(\begin{array}{cc}2 & 1 \\ 1 & 2\end{array}\right)$

To obtain the system of equations including all nodes, these element matrices must be assembled that its resulting system has the following form,

$A \frac{\partial u}{\partial r}+[\mu B+v C] u=0$

By applying Crank-Nicolson approach and substituting $u=\frac{u^{n}+u^{n+1}}{2}$ and $u_{r}=\frac{u^{n}-u^{n+1}}{\Delta r}$ into equation (17), the $N_{z} \times N_{z}$ tridiagonal matrix system obtained,

$$
\begin{aligned}
& {\left[A+\frac{\Delta r}{2}(\mu B+v C)\right] u^{n+1}} \\
& =\left[A-\frac{\Delta r}{2}(\mu B+v C)\right] u^{n}
\end{aligned}
$$

Where, $\Delta r$ is the range step. By doing all of above steps Galerkin finite element parabolic equation method is derived.

\section{Numerical Results}

In this section, the FEPE technique is applied for solving six test problems. First problem, as shown in figure 1 , just shows comparison of transmission loss (TL) curve for different receiver depths. However, simulation result in figure 2 shows the impact of multilayer media in FEPE. For the third example shown in figure 3 , obtained results are compared with standard UMPE model [11]. The third example also represents a brief comparison of method with standard KRAKEN normal mode technique [12]. In all implementations, for maintaining reflection of bottom interface, we use an artificial absorbing layer. Second test problem includes environment with final depth $800 \mathrm{~m}$ sloping wedge with penetrable lossy bottom. The initial water depth is $500 \mathrm{~m}$ decreasing linearly with slopes upward at approximately $1.7^{\circ}$. The final range of simulation is $4 \mathrm{~km}$. In water layer sound velocity and density are $c_{w}=1500 \mathrm{~m} / \mathrm{s}$ and $\rho_{w}=1 \mathrm{~g} / \mathrm{cm}^{3}$. Source with frequency $25 \mathrm{~Hz}$ locates at depth $100 \mathrm{~m}$ (figure 3). In elastic lossy sediment, speed, density and attenuation are $c_{b o t}=1700 \mathrm{~m} / \mathrm{s}$, $\rho_{\text {bot }}=1.5 \mathrm{~g} / \mathrm{cm}^{3} \quad$ and $\alpha_{\text {bot }}=0.5 \mathrm{~dB} / \mathrm{km}$, respectively.

The depth and range segments are chosen 500 and 500. Figure 4, shows TL in the receiver depth of $30 \mathrm{~m}$. The range of simulation for all plots of this example is $5 \mathrm{~km}$. This example involves other case for comparison of impact of thickness of bottom layer on water column propagation.

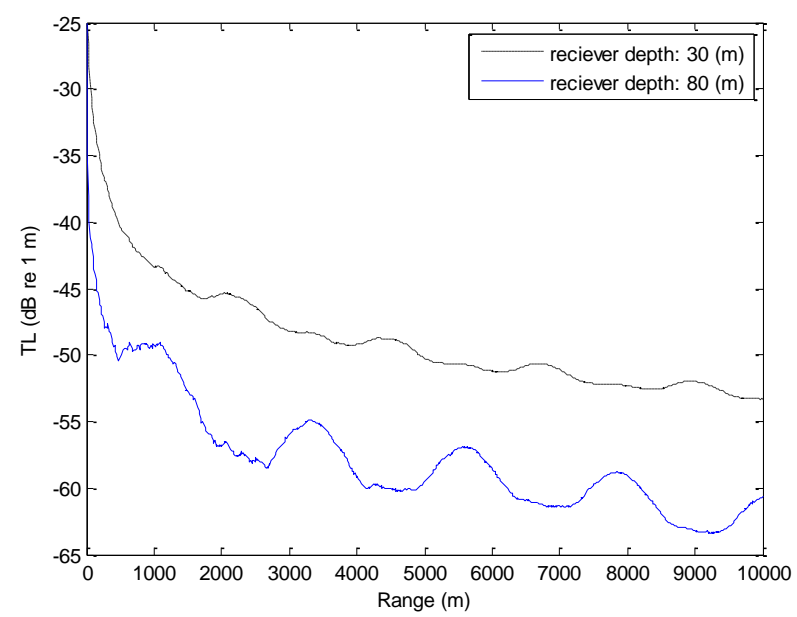

Figure. 1 The comparison of TL curves for two different receiver depths. 


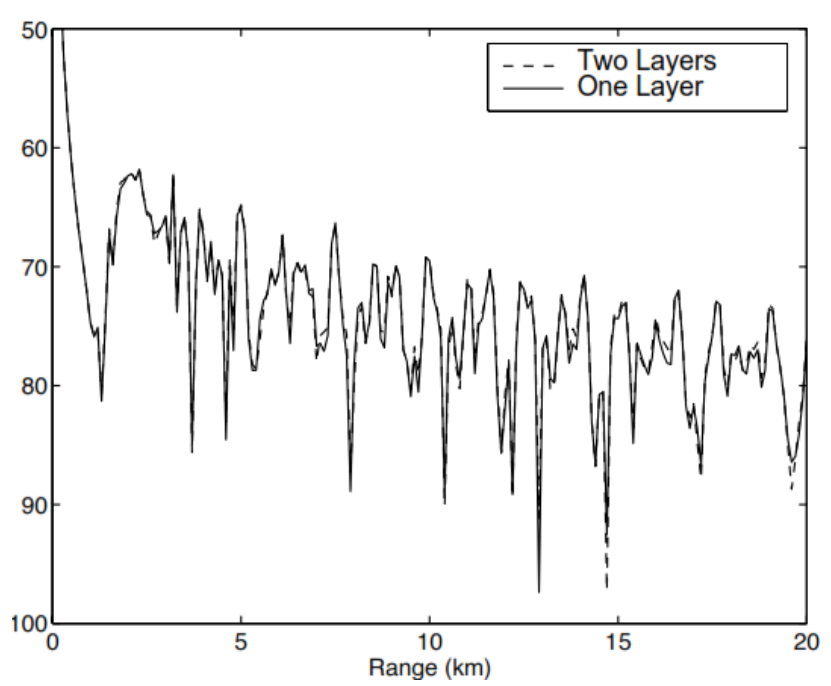

Figure. 2 Comparison of one bottom layer and two layers environment in water media.

In the TL curve plot (Figure 2) and pressure field (Figure 3), the significant differences of the water column at long range illustrate the influence of sediment thickness on the acoustic pressure field.
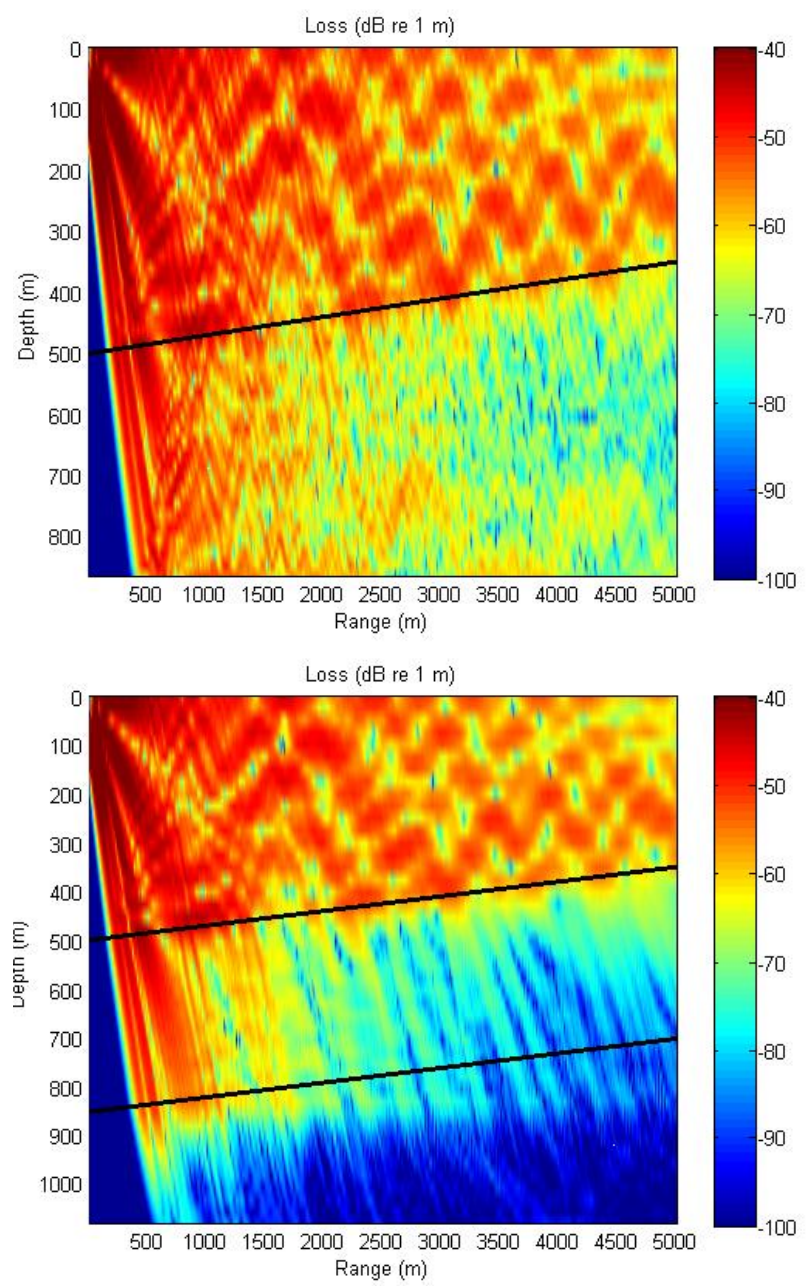

Figure. 3 The acoustic pressure field for an environment with two different bottom thicknesses
Third problem consists of a water column with variable bottom topography ranged from $400 \mathrm{~m}$ to $250 \mathrm{~m}$ on top of sediment with compression speed, density and attenuation similar to those of mentioned in second test problem. The source depth is set to $100 \mathrm{~m}$ and the source frequency is set 100 Hz. Figure 4 shows the acoustic pressure field of this problem for final rang of simulation is $10 \mathrm{~km}$. To highlight the performance of the present method, we compare the obtained result of FEPE with the simulation result of UMPE standard software [7]. Figure 5 shows TL curve comparison that using the same environmental properties such as type of initial field, sampling grid points in both depth and depth, attenuation mechanism, absorption layer structure and shear property of bottom interface parameters (speed, density and attenuation) can give results that are quite close to those of obtained by running UMPE.

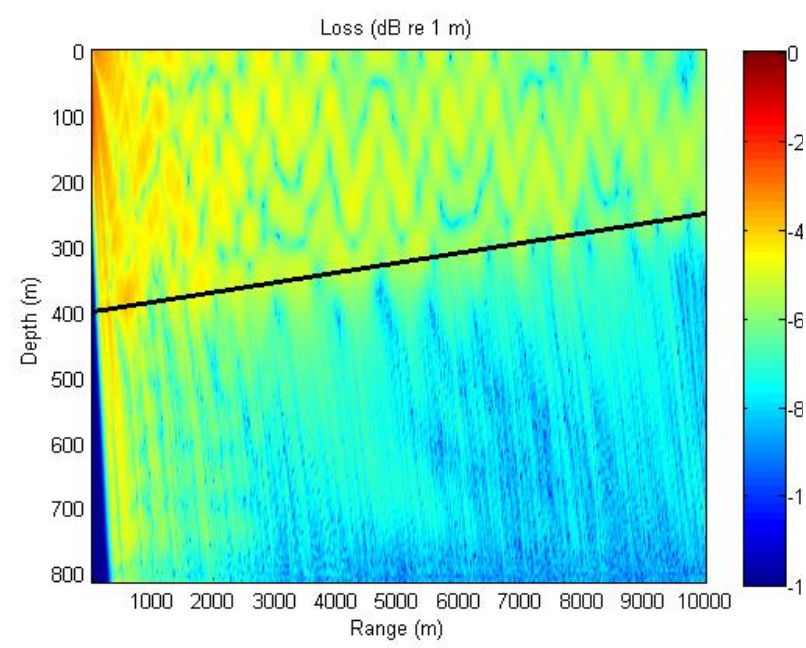

Figure. 4 The acoustic pressure field for third test case

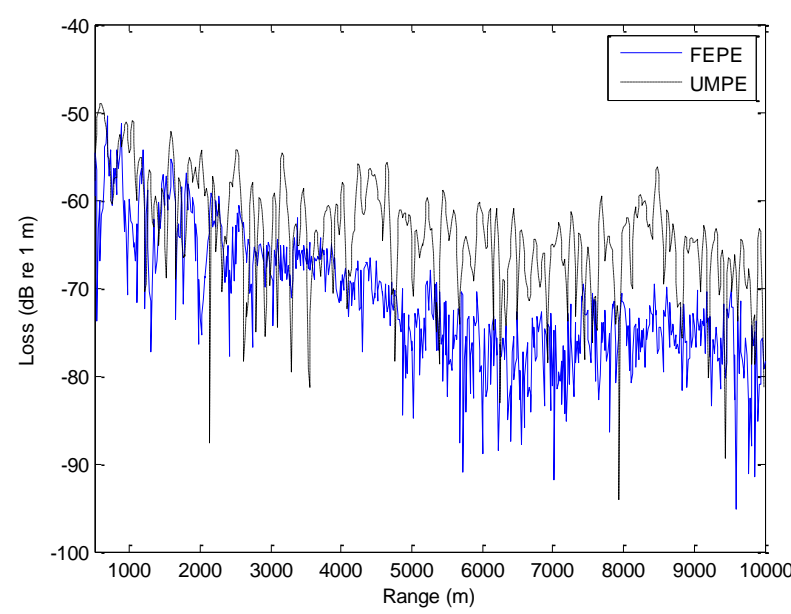

Figure. 5 The comparison of standard UMPE method with FEPE for the same initial set points. 


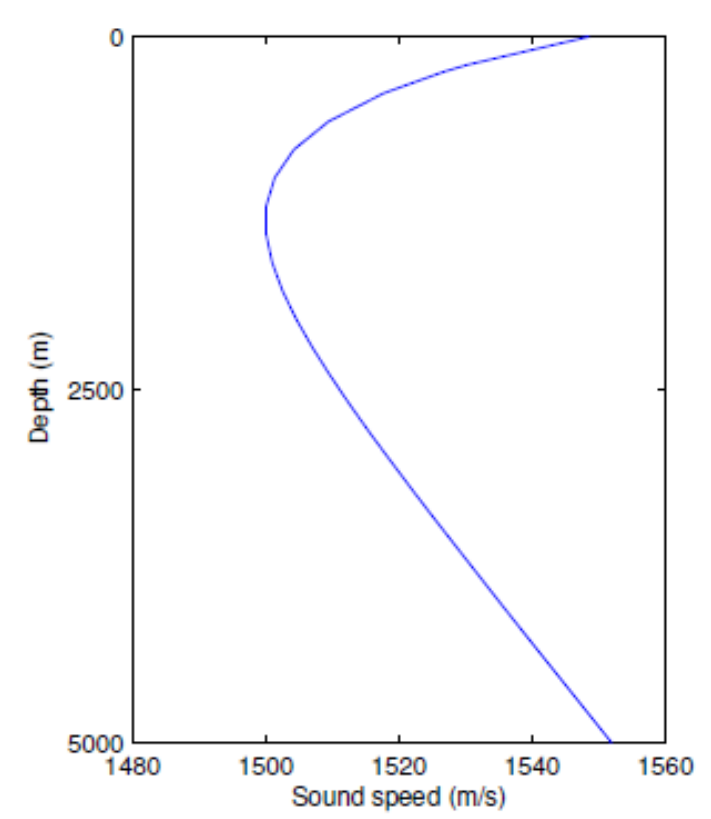

Figure. 6 The Munk sound speed profile of deep water.

The last environment is a deep ocean including water column of depth $5000 \mathrm{~m}$ and the Munk sound speed profile is given in figure 6 . The final simulation range is set $50 \mathrm{~km}$ for a source depth of $91.6 \mathrm{~m}$, a receiver depth of $131 \mathrm{~m}$ and source frequency of $20 \mathrm{~Hz}$. Figure 7 shows comparison of TL curves of standard Keraken normal mode method [12] and proposed technique. As can be seen from this figure, the proposed method has good agreement with the Keraken normal mode.

To investigate the capability of the method presented in this study, we introduce a waveguide problem in which the fluid half-space with greater of $100 \mathrm{~m}$ and with values of speed and absorption given by $500 \mathrm{~m} / \mathrm{s}$ and $1 \mathrm{~dB} / \lambda$, respectively. TL comparisons in the waveguide are shown in Figure 8. As can be seen, the proposed method result has a good agreement with the UMPE result.

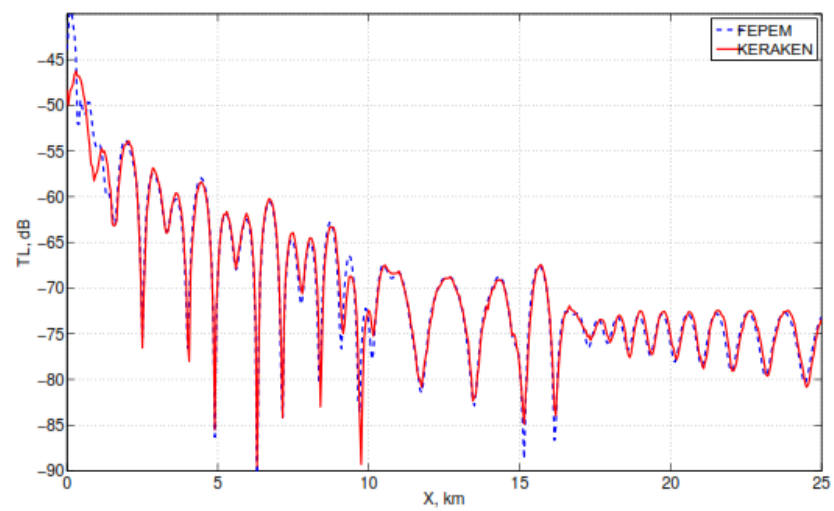

Figure. 7 The comparison of KRAKEN normal mode and FEPE method for similar environmental parameters.
In this paper, propagation of low frequency sound speed in underwater was investigated. To simulate this underwater acoustic phenomenon, parabolic equation method is applied and the governing equations are numerically solved by implicit finite difference scheme. Two benchmark problems are used for the validation of the developed method for the range dependent problems. The obtained numerical results are compared against the reported results of semi-analytical methods and good agreement is displayed.

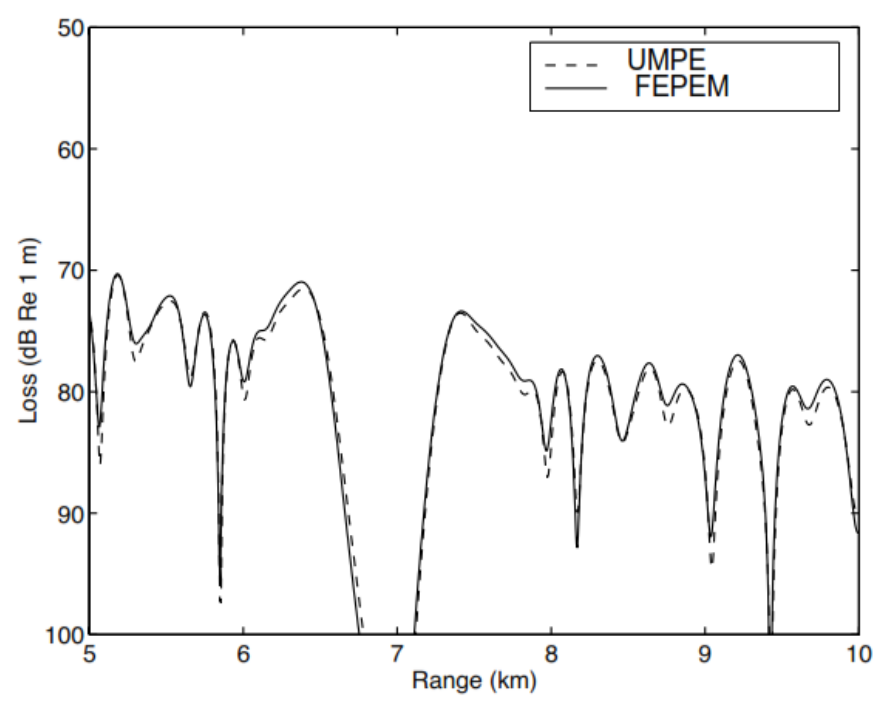

Figure. 8 Transmission loss comparisons for the solid bottom. The source and receiver depths are $100 \mathrm{~m}$.

\section{Conclusions}

Sound propagation at low frequency is one of the most important subjects of research in underwater acoustics. In the paper, parabolic equation method was applied for numerically analyzing sound propagation with low frequencies in different situations. To accomplish this task, Galerkin finite element approach was adopted to solve the governing parabolic equation. All the analyses and numerical investigations conducted in this paper were accomplished by a developed computer program which was written in MATLAB software.

The capability of proposed method was validated by comparing transmission loss predictions to predictions obtained using standard benchmark models for several test cases. For validation of proposed method, we chose to compare our results to those obtained using UMPE. The numerical results demonstrated good agreement in comparison with physically behavior of wave propagation in ocean environment. Moreover, proposed method was proved as a reliable method for wide angle, 
bottom-interacting situation with complex boundaries in ocean acoustic wave propagation. 2nd International Conference and Exhibition on Underwater Acoustics, June 2014, pp. 1-7, 2014.

\section{References}

[1] M. D. Collins, W.A. Kuperman b, B. Edward McDonaldc and W. L.Siegmann, "Parabolic equation modeling of azimuthally advected gravity waves", Wave Motion, vol. 31, pp. 131-138, 2000.

[2] W. Luo and H. Schmidt, "Three-dimensional propagation and scattering around a conical seamount," J. Acoust. Soc. Am., vol. 125, pp. 52-65, 2009.

[3] Y. T. Lin, J. M. Collis, T. F. Duda, "A threedimensional parabolic equation model of sound propagationbusing higher-order operator splitting and pade approximants", J. Acoust. Soc. Am., vol. 132, Issue 5, pp. 364-370, 2012.

[4] J. M. Collisa, W. L. Siegmann, F. B. Jensen, M. Zampolli, E. T.Küsel, M. D. Collins, "Parabolic equation solution of seismo-acoustics problems involving variations in bathymetry and sediment thickness", J. Acoust. Soc. Am., vol. 123, Issue 1, pp. 51-55, 2008.

[5] M. Bakhoday Paskyabi, F. Rashidi, Wide Angle Parabolic Equation Based on One-Periodic Daubechies Wavelet for Modelling Underwater Wave Propagation, in WSEAS Trans. on Math., vol. 4, pp. 204-211, 2005.

[6] Y.-T. Lin, T.F. Duda, C. Emerson, G.G. Gawarkiewicz, A.E. Newhall, B. Calder, J.F. Lynch, P. Abbot, Y.-J. Yang and S. Jan, "Experimental and numerical studies of sound propagation over a submarine canyon northeast of Taiwan," IEEE $J$. Ocean. Eng., vol. 40, pp. 237-249, 2015.

[7] A.A. Shmelev, J.F. Lynch, Y.-T. Lin and H. Schmidt, "3D coupled mode analysis of internal-wave acoustic ducts," J. Acoust. Soc. Am., vol. 135, pp. 2497-2512, 2104.

[8] L.Y.S. Chiu, A. Chang, Y.-T. Lin, and C.-S. Liu, "Estimating Geo-acoustic Properties of the Surficial Sediments in the Region of North Mein-Hua Canyon with a Chirp Sonar Profiler," IEEE J. Ocean. Eng., vol. 40, Issue 1, pp. 222-236, 2015.

[9] Y.-T. Lin, "Numerical modeling of three-dimensional underwater sound propagation under rough sea surfaces," J. Acoust. Soc. Am., vol. 135, pp. 24-29, 2014

[10] Y.-T. Lin, "A higher-order tangent linear parabolicequation solution of three-dimensional sound propagation," J. Acoust. Soc. Am., vol. 134, pp. 251257, 2013.

[11] Y.-T. Lin, W. G. Zhang, and T. F. Duda, "Sensitivity of the underwater sound field in submarine canyons to water column variability," J. Acoust. Soc. Am., vol. 134, pp. 4114, 2013.

[12] Y.-T. Lin, "Numerical applications of a higher order square-root Helmholtz operator splitting method on modeling three-dimensional sound propagation," in: 